\title{
The Impact of Hepatitis Virus Infection on Acute Pancreatitis: A Population-Based Study the Impact of Acute Pancreatitis in Patients with Known Hepatitis Virus Infection
}

\author{
Lei Li* \\ Department of Gastroenterology and Hepatology, Zhongshan Hospital, Fudan University, China \\ *Corresponding author: Lei Li, Department of Gastroenterology, Zhongshan Hospital, Fudan University, China \\ To Cite This Article: Lei Li. The Impact of Hepatitis Virus Infection on Acute Pancreatitis: A Population-Based Study the Impact of Acute \\ Pancreatitis in Patients with Known Hepatitis Virus Infection. Am J Biomed Sci \& Res. 2021 - 13(4). AJBSR.MS.ID.001897. \\ DOI: 10.34297/AJBSR.2021.13.001897.
}

Received: 眥 July 06, 2021; Published: 䟧 July 22, 2021

\begin{abstract}
Background and study Aim: We investigated associations between hepatitis virus infection and outcomes of Acute Pancreatitis (AP), and the impact of hepatitis virus subtypes and effects of liver transplantation on AP outcomes in hepatitis virus-infected AP patients.

Patients and methods: In this cross-sectional study, data of 163,140 AP patients (5,599 with viral hepatitis; 157,541 without) were extracted from the 2005-2014 Nationwide Inpatient Sample database and analyzed retrospectively using logistic regression. Primary outcomes were inhospital mortality, leaving Against Medical Advice (AMA), and other diagnoses (i.e., colonic necrosis, splenic vein/portal vein thrombosis, and organ failure). Secondary outcome was hospital length of stay.

Results: AP patients with hepatitis virus infection had increased odds of death or AMA $(\mathrm{aOR}=1.39,95 \% \mathrm{CI}=1.23-1.57$, $\mathrm{p}<0.001)$ and organ failure (OR=1.19, 95\% CI=1.07-1.33, $\mathrm{p}<0.001)$. A significant correlation was shown between death or AMA and liver transplantation. Illness severity was significantly different between hepatitis virus subtypes $(\mathrm{p}<0.001)$; the majority of HAV and HBV patients had major loss of function $(42.44 \%$ and $48.44 \%$, respectively and $48.87 \%$ of $\mathrm{HCV}$ patients had moderate loss of function.

Conclusions: AP patients with hepatitis virus infection have greater odds of death or AMA and organ failure than those without viral hepatitis, and liver transplantation increases odds for death and AMA but not for organ failure. HCV occurs more frequently in AP patients but illness is more severe with HAV and HVB. The results of the present study may help develop strategies targeting anti-viral agents to ameliorate AP in hepatitis virus-infected patients.
\end{abstract}

Keywords: Acute Pancreatitis; Hepatitis Virus; Infection; Outcomes; National Inpatient Sample (NIS); HCV; Organ Failure; Anti-Viral Agents; Leptospira; Mycoplasma; Hypertriglyceridemia

\section{Introduction}

Acute Pancreatitis (AP) is inflammation of the pancreas, characterized clinically by acute abdominal pain and elevated levels of pancreatic enzymes in the blood. Incidence is about 36.4 in 100,000 population annually, with women affected more than men (7.9\% vs. 5.7\%, respectively); incidence of AP is increasing in the US and Europe, making it the leading cause of hospitalization due to gastrointestinal disorders [1]. Gallstones (40\%-70\%) and excess or prolonged alcohol consumption (30\%) are the most frequent causes of AP, followed by hypertriglyceridemia [2], certain drugs (e.g., azathioprine, valproic acid, ACE-inhibitors) [3], infections, trauma and gene mutations and polymorphisms [2,4]. In about $10 \%$ of AP cases, miscellaneous causes are responsible, including infection by parasites (e.g., Ascaris lumbricoides), bacteria (e.g., Leptospira, Mycoplasma), and viruses (e.g., mumps-virus, herpes simplex, varicella-zoster, coxsackievirus and hepatitis viruses) [5], with each organism causing AP through different mechanisms [4]. However, to date, studies on the viral causes of AP are limited. 
More than half of hepatitis cases in the United States are caused by the presence of a specific hepatitis virus. The most common causes are Hepatitis A Virus (HAV), Hepatitis B Virus (HBV) and Hepatitis C Virus (HCV), any of which may result in acute disease with symptoms such as nausea, abdominal pain, fatigue, and jaundice, leading to significant morbidity and mortality worldwide [6]. Acute infection may lead to chronic infection and sequelae, sometimes progressing to cirrhosis and Hepatocellular Carcinoma (HCC) [6,7]. Hepatitis D Virus (HDV) and Hepatitis E Virus (HEV) have been found to cause hepatitis sporadically, primarily in countries where the viruses are endemic [7]. The surveillance report of the Centers for Disease Control and Prevention (CDC) reported incidence of HAV (0.4 per 100,000 population), HBV (1.1 per 100,000 population) and HCV (0.8 per 100,000 population) in 2015 , but the absence of symptoms in many cases precludes accurate incidence [8].

While hepatitis viruses infect mainly hepatocytes, tissues of other organs such as the pancreas have also been found to carry antigens for hepatitis viruses A, B, C, and non-A/non-B viruses [9]. $\mathrm{AP}$ is associated with fulminant hepatic failure and hepatitis virus antigens have been found in results of histological and serological examination of pancreatic tissue at autopsy [10]. A study of the frequency and characteristics of pancreatitis in acute viral hepatitis found that AP occurs in 5.65\% of non-fulminant acute hepatitisinfected patients and that it is usually mild and both diseases can be managed conservatively; among 124 patients, AP was associated with HAV in two patients, HBV in one patient and HEV in 4 patients [11]. Given the known associations between viral hepatitis and AP, we hypothesized that hepatitis virus infection may have an impact on AP outcomes, and that different hepatitis virus subtypes and liver transplantation may influence AP outcomes to some degree. Ultimately, such findings relative to hepatitis virus subtypes may add insight into AP outcomes and help to develop strategies to treat hepatitis virus-infected AP patients. Therefore, this study aimed to investigate associations between hepatitis virus infection and outcomes of AP, and to explore the frequency and outcomes of AP associated with various hepatitis subtypes as well as the effects of liver transplantation on the outcomes of AP patients with hepatitis virus infection.

AP related to fulminant viral hepatitis has been known, and the association of AP with non-fulminant viral hepatitis is rare. The mortality of AP relies on the severity of hepatitis rather than pancreatitis. Usually, these cases are attributed to HAV, HBV, HCV or HEV [12]. In patients with known hepatitis virus infection, AP may be induced during the therapeutic process. Choi et al. reported that AP may occur during the combination therapy of PEG-IFN- $\alpha-2 \mathrm{a}$ and
RBV for chronic hepatitis C; thus, it is necessary to stop medical administration if AP resulted from pegylated interferon-alpha $2 \mathrm{a}$ (PEG-IFN- $\alpha-2 \mathrm{a}$ ) and Ribavirin (RBV) was suspected [13]. Ando et al. demonstrated that PEG-IFN $\alpha$ and RBV combination therapy is a potential cause of drug-induced AP in patients with Chronic Hepatitis C (CHC) [14]. Therefore, this study aimed to investigate associations between hepatitis virus infection and outcomes of AP, and to explore the frequency and outcomes of AP associated with various hepatitis subtypes as well as the effects of liver transplantation on the outcomes of AP patients with hepatitis virus infection.

\section{Patients and Methods}

\section{Data source}

Data for this study were extracted from the 2005-2014 Nationwide Inpatient Sample (NIS), a database developed by the Healthcare Cost and Utilization Project (HCUP) in the United States that is maintained by the Agency for Healthcare Research and Quality (AHRQ) [12,15]. The NIS database represents a $20 \%$ sample of inpatient admissions from 45states and 1051 hospitals that participated in collecting patient data at discharge. Principal and secondary diagnoses, principal and secondary procedures, admission date and diagnosis, discharge status, patient demographic data, and length of stay are included for each inpatient. Statistical weights that allow generalized estimates of national case volumes are also provided in the NIS.

Study design and ethical considerations. A cross-sectional, population-based retrospective study was conducted to analyze inpatient data collected at patient discharge by the HCUP-NIS administrative database for ten years from 2005 to 2014. The certificate number HCUP-6FVS78J20 was obtained from HCUP-NIS. The study conforms to the data-use agreement for the NIS from HCUP $[12,15]$. Because the NIS originally received signed informed consent from all included patients to participate in data collection for later evaluation, and patient data in the NIS database were subsequently deidentified, signed informed consent was waived for the present study.

\section{Study population}

Among 549,736 eligible patients from the 2005-2014 NIS database with the diagnosis of acute pancreatitis (AP) (ICD-9-CM 577.0), data of 163,400 patients with AP were included. Patients with other significant causes of AP that could act as confounders to the effects of hepatitis were excluded, including those with gall stones (ICD-9-CM 560.31), alcohol abuse (ALCOHOL/Severity), and lipid metabolism disorders (ICD-9-CM272). 


\section{Main outcomes and variables}

Primary and secondary outcomes: The primary outcomes were in-hospital mortality (DIED=1) or discharged against medical advice (AMA)(DISPUNIFORM7, 20/ Core); and other diagnoses: colonic necrosis (ICD-9-CM557); splenic vein thrombosis/ portal vein thrombosis (ICD-9-CM289.59, 452); organ failure, including respiratory (ICD-9-CM518.81); renal (ICD-9-CM586, V56); cardiovascular (ICD-9-CM428 ); hepatic (ICD-9-CM570); coagulation (ICD-9-CM286.7, 286.) or neurologic (ICD-9CM780.01). The secondary outcome was length of stay (LOS).

Organ failure definitions: Organ failure was defined by laboratory values or other measurements as follows: Respiratory failure was defined by PaO2/FIO2 $<300$ or use of mechanical ventilator. Renal failure was defined by serum creatinine $\geq 2.0 \mathrm{mg} /$ dl. Cardiovascular organ failure was defined by systolic blood pressure $<90 \mathrm{mmHg}$ or the use of vasopressor. Coagulation system failure was defined by platelet count $<100$ 103/mm3. Liver failure was defined by bilirubin $\geq 2.0 \mathrm{mg} / \mathrm{dL}$. Neurologic failure was defined by Glasgow Coma Score $<10$.

Subjects' demographic characteristics: Demographic variables included patient age, gender, and ethnicity (grouped into white, black, Hispanic, others). Comorbidity measures applied here are obesity, Chronic Kidney Disease (CKD), solid tumor without metastasis, and metabolic syndrome.

Severity of illness: The severity of illness of AP patients with hepatitis virus infection was determined based on the diagnostic code used by HCUP/NIS, All Patient Refined-Disease Related Groups represented by the code APR-DRG_Severity/Severity. Severity of illness was classified into four groups according to APRDRG ranking as: minor loss of function, moderate loss of function, major loss of function, and extreme loss of function.

\section{Statistical analysis}

Categorical variables were summarized as frequency and percentage and were assessed using the $\chi 2$ test. Numerical variables were presented as mean and Standardized Error (SE) and median with Interquartile Rang (IQR). Logistic regression analysis was performed to estimate associations between hepatitis virus and death or discharged Against Medical Advice (AMA); or with secondary diagnosis of colonic necrosis, splenic vein thrombosis/ portal vein thrombosis, or organ failure. The linear regression model was used to assess the association between hepatitis virus and Length of Stay (LOS). Significant variables having a P value of $<0.05$ in univariate analysis were analyzed using multivariate analysis, and other variables (age, gender, race, obesity, CKD, solid tumor without metastasis, and metabolic syndrome) were also evaluated by multivariate analysis with stepwise selection. All $\mathrm{P}$ values were two-sided and $\mathrm{P}<0.05$ was established as statistical significance. Considering the complex sampling design of the HCUP-NIS data, all statistical analyses were performed using PROC SURVEY procedures in the statistical software package SAS software version 9.4 (SAS Institute Inc., Cary, NC, USA). All results reported are weighted unless noted otherwise.

\section{Results}

\section{Study population}

A total of 549,736 (weighted $n=2,716,399$ ) patients with the coded diagnosis of Acute Pancreatitis (AP) were identified in the 2005-2014 NIS database. After excluding patients with gall stones, alcohol abuse, and disorders of lipid metabolism, 195,894 (weighted $\mathrm{n}=967,979$ ) AP patients remained. After excluding missing data for LOS, age, gender, race, and hospital bed count (representing hospital size), the data of 163,140 patients comprised the analytic sample.

\section{Subjects' demographic and clinical characteristics}

The final study population of 163,140 subjects (weighted $n=806,579$ ) included 5,599 subjects (weighted $n=27,631 ; 3.4 \%$ ) with hepatitis virus infection and 157,541 (weighted $n=778,948$; $96.6 \%$ ) without hepatitis virus infection. Mean age of included subjects was 50.3 years; $43.6 \%$ were males and $56.4 \%$ females, and most subjects $(65.5 \%)$ were White race. Clinical characteristics included obesity 8.93\%, Chronic Kidney Disease (CKD) 8.06\%, solid tumor without metastasis $1.44 \%$, and metabolic syndrome $0.13 \%$. Among the 5,599 hepatitis-infected AP patients, 4921 (87.89\%) of patients were infected with HCV (Table 1).

\begin{tabular}{|c|c|}
\hline Demography & Overall N (wt \%) \\
\hline \multicolumn{2}{|c|}{ Age } \\
\hline Mean \pm SE & $50.32 \pm 0.05$ \\
\hline Median (IQR) & $49.31(36.30,62.76)$ \\
\hline \multicolumn{2}{|c|}{ Gender } \\
\hline Male & $71136(43.64)$ \\
\hline Female & 92004 (56.36) \\
\hline
\end{tabular}




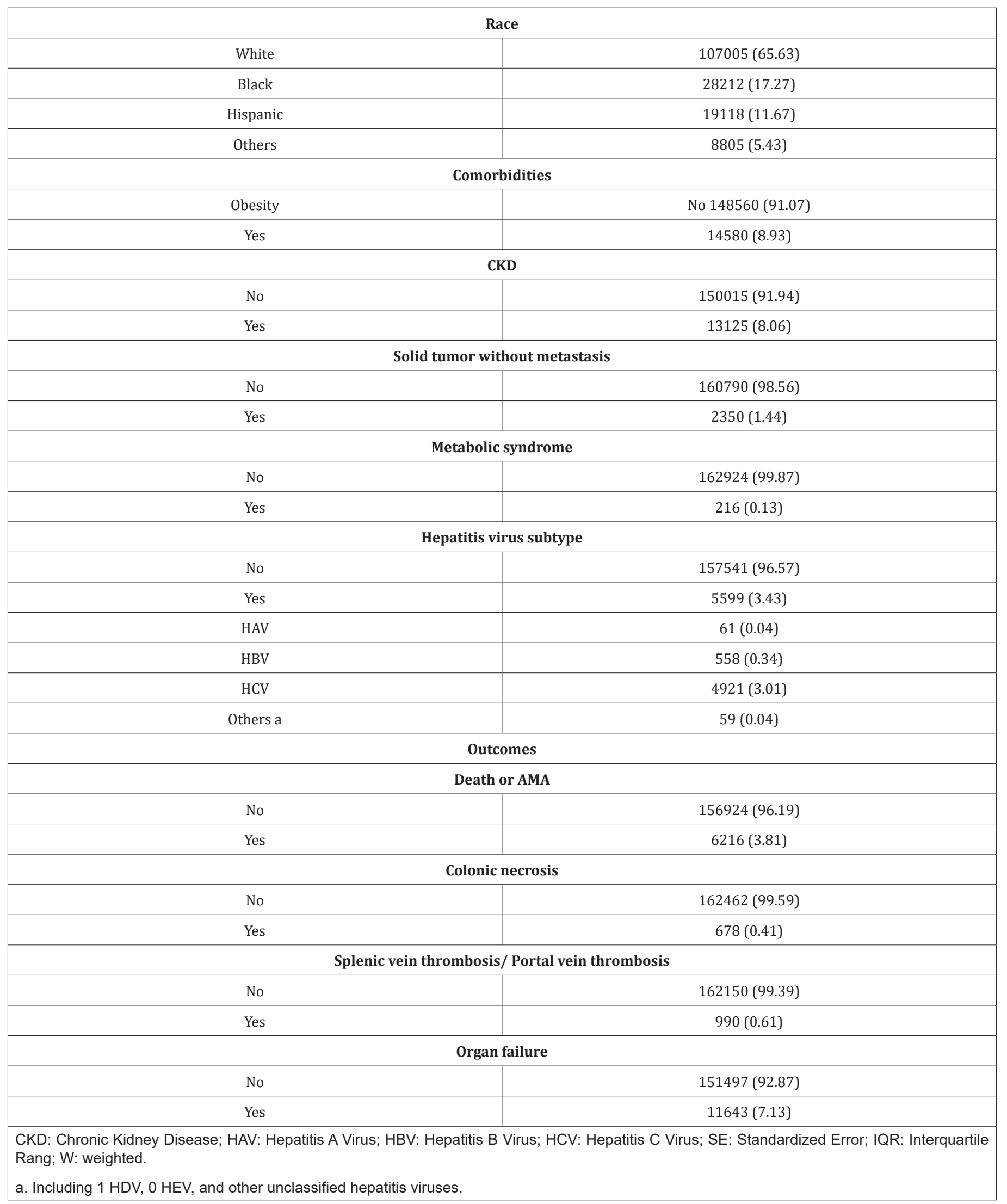




\section{Univariate and multivariate logistic regression analyses}

Univariate analysis revealed that AP patients with hepatitis virus were significantly associated with increased odds of death or leaving hospital AMA (OR=1.60, 95\% CI=1.42-1.80, $\mathrm{p}<0.001$ ) and organ failure $(\mathrm{OR}=1.16,95 \% \mathrm{CI}=1.05-1.29, \mathrm{p}=0.004$; Table 2). After adjusting for variables by stepwise model selection, results of multivariate regression analysis showed that subjects with hepatitis virus infection were still significantly associated with increased odds of death or AMA (aOR=1.39, 95\% CI=1.23-1.57, $\mathrm{p}<0.001)$ and organ failure $(\mathrm{OR}=1.19,95 \% \mathrm{CI}=1.07-1.33, \mathrm{p}<0.001$;
Table 2). However, univariate analyses revelated that hepatitis virus infection was not significantly associated with colonic necrosis, splenic vein thrombosis/ portal vein thrombosis, or LOS (Table2 \& 3). Multivariate analysis revealed that compared to AP patients without hepatitis virus infection, only AP patients with HCV had significantly higher odds of death or AMA positive $(\mathrm{aOR}=1.42$, 95\% CI=1.25-1.61, $\mathrm{p}<0.001$ ) (Table 4). In addition, significantly increased odds of organ failure were found in patients with HBV (aOR=1.46, 95\% CI=1.08-1.96, $\mathrm{p}=0.013$;) and HCV (aOR=1.16, 95\% $\mathrm{CI}=1.03-1.31, \mathrm{p}=0.013$ ) (Table 5).

\begin{tabular}{|c|c|c|c|c|c|}
\hline Outcomes & $\mathrm{N}(\mathrm{wt} \%)$ & OR $(95 \% \mathrm{CI})$ & p-value & aOR $(95 \% \mathrm{CI})$ & p-value \\
\hline \multicolumn{6}{|l|}{ Death or AMA } \\
\hline Without hepatitis virus & $5890(3.74)$ & reference & & reference & \\
\hline With hepatitis virus & $326(5.83)$ & $1.60(1.42,1.80)$ & $<0.001$ & $1.39(1.23,1.57)^{\mathrm{a}}$ & $<0.001$ \\
\hline \multicolumn{6}{|l|}{ Colonic necrosis } \\
\hline Without hepatitis virus & $657(0.42)$ & reference & & & \\
\hline With hepatitis virus & $21(0.38)$ & $0.91(0.59,1.40)$ & 0.653 & & \\
\hline \multicolumn{6}{|l|}{$\begin{array}{l}\text { Splenic vein thrombosis/ } \\
\text { Portal vein thrombosis }\end{array}$} \\
\hline Without hepatitis virus & $951(0.60)$ & reference & & & \\
\hline With hepatitis virus & $39(0.71)$ & $1.18(0.84,1.64)$ & 0.335 & & \\
\hline \multicolumn{6}{|l|}{ Organ failure } \\
\hline Without hepatitis virus & $11184(7.09)$ & reference & & reference & \\
\hline With hepatitis virus & $459(8.16)$ & $1.16(1.05,1.29)$ & 0.004 & $1.19(1.07,1.33)^{\mathrm{a}}$ & $<0.001$ \\
\hline
\end{tabular}

Table 3: Association between hospital length of stay and hepatitis virus.

\begin{tabular}{|c|c|c|c|c|}
\hline & & & \multicolumn{2}{c|}{ LOS } \\
\hline & N (wt \%) & Mean \pm SE & & \\
\hline Hepatitis virus & & & reference \\
\hline No & $151651(96.65)$ & $4.97 \pm 0.02$ & -0.06 \\
\hline Yes & $5273(3.35)$ & $4.90 \pm 0.07$ & 0.398 \\
\hline
\end{tabular}

Table 4: Associations between hepatitis virus subtypes and death or AMA in subjects with acute pancreatitis.

\begin{tabular}{|c|c|c|c|c|c|c|c|}
\hline \multicolumn{8}{|c|}{ Death or AMA } \\
\hline \multirow{3}{*}{\multicolumn{2}{|c|}{ Overall }} & No & Yes & \multirow{4}{*}{ OR $(95 \% \mathrm{CI})$} & \multirow{4}{*}{ p-value } & \multirow{4}{*}{$\operatorname{aOR}(95 \% \mathrm{CI})^{\mathrm{b}}$} & \multirow{4}{*}{ p-value } \\
\hline & & $n=156924$ & $n=6216$ & & & & \\
\hline & & $\begin{array}{c}\text { weighted } \mathbf{n}= \\
775869\end{array}$ & $\begin{array}{c}\text { weighted } \mathbf{n}= \\
30710\end{array}$ & & & & \\
\hline & N (wt \%) & N (wt \%) & N (wt \%) & & & & \\
\hline \multicolumn{8}{|c|}{ Hepatitis virus } \\
\hline No & 157541 (96.57) & 151651 (96.65) & $5890(94.75)$ & reference & & reference & \\
\hline HAV & $61(0.04)$ & $58(0.04)$ & $3(0.05)$ & $1.33(0.42,4.27)$ & 0.628 & $1.36(0.43,4.34)$ & 0.599 \\
\hline HBV & $558(0.34)$ & $530(0.34)$ & $28(0.46)$ & $1.40(0.96,2.05)$ & 0.084 & $1.14(0.78,1.67)$ & 0.503 \\
\hline $\mathrm{HCV}$ & $4921(3.01)$ & $4630(2.95)$ & $291(4.68)$ & $1.62(1.43,1.83)$ & $<0.001$ & $1.42(1.25,1.61)$ & $<0.001$ \\
\hline Others & $59(0.04)$ & $55(0.04)$ & $4(0.06)$ & $1.80(0.64,5.05)$ & 0.261 & $1.94(0.70,5.40)$ & 0.203 \\
\hline
\end{tabular}


AMA: Against Medical Advice; HAV: Hepatitis A Virus; HBV: Hepatitis B Virus; HC: Hepatitis C Virus; OR: Odds Ratio; AOR: Adjusted Odds Ratio; Cl: Confidence Interval; WT: weighted.

a. Included $1 \mathrm{HDV}, 0 \mathrm{HEV}$, and other hepatitis virus that cannot be classified in the dataset.

b. Model was adjusted by age, gender, race, obesity, and CKD. Significant values in bold $(P<0.05)$.

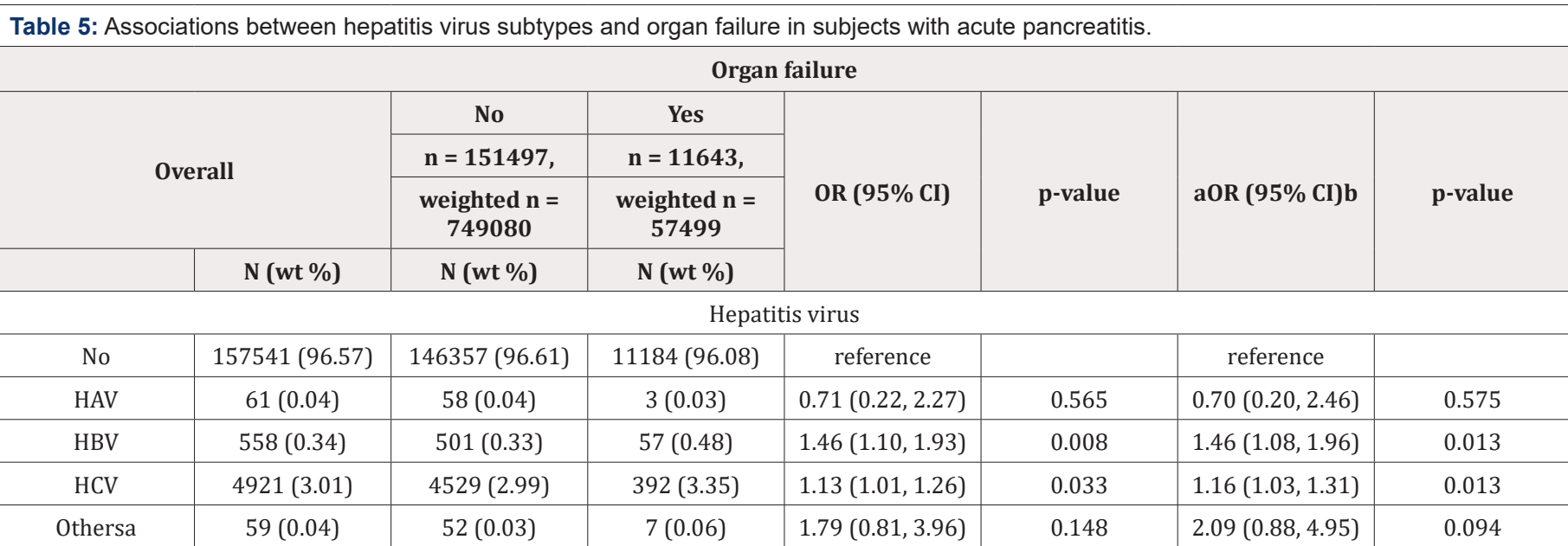

HAV: Hepatitis A Virus; HBV: Hepatitis B Virus; HCV: Hepatitis C Virus; OR: Odds Ratio; AOR: Adjusted Odds Ratio; Cl: Confidence Interval; WT: Weighted.

a. Including $1 \mathrm{HDV}, 0 \mathrm{HEV}$, and other unclassified hepatitis viruses b Model was adjusted for age, gender, race, obesity, and CKD. Significant values in bold $(\mathrm{P}<0.05)$

Among 5599 subjects with hepatitis virus infection, 73 had undergone liver transplantation, including one with HAV, 7 with HBV, and 65 with HCV (Table 6). Significant differences were found in severity of illness between the subtypes of hepatitis virus $(\mathrm{p}<0.001)$. Most patients with HAV and HBV had major loss of function (42.44\% and $48.44 \%$, respectively). Most patients with HCV and other hepatitis viruses had moderate loss of function $(48.87 \%$ and $51.77 \%$, respectively) (Table 6). Significant differences were found in the number of deaths or AMA between hepatitis virus-infected subjects with liver transplantation compared to those without ( $p=0.021)$, although only one subject with liver transplantation in this cohort died or left the hospital AMA. However, no significant differences were found in organ failure between hepatitis virusinfected patients with or without liver transplantation $(p=0.291)$ (Table 7).

\begin{tabular}{|c|c|c|c|c|c|}
\hline & HAV & HBV & HCV & Others a & p-value \\
\hline & N (wt \%) & N (wt \%) & N (wt \%) & N (wt \%) & \\
\hline Liver transplantation & & & & & NA \\
\hline No & $60(98.20)$ & 551 (98.69) & 4856 (98.67) & $59(100)$ & \\
\hline Yes & $1(1.80)$ & $7(1.31)$ & $65(1.33)$ & $0(0)$ & \\
\hline $\begin{array}{l}\text { Severity of illness Minor loss of function } \\
\text { (includes cases with no comorbidity or } \\
\text { complications) }\end{array}$ & $8(13.54)$ & $14(2.56)$ & $301(6.19)$ & $12(20.55)$ & $<0.001$ \\
\hline Moderate loss of function & $24(39.27)$ & $226(40.63)$ & $2401(48.87)$ & $31(51.77)$ & \\
\hline Major loss of function & $26(42.44)$ & $271(48.44)$ & $1882(38.1)$ & $11(18.84)$ & \\
\hline Extreme loss of function & $3(4.74)$ & 47 (8.37) & $337(6.83)$ & $5(8.84)$ & \\
\hline
\end{tabular}




\begin{tabular}{|c|c|c|c|c|}
\hline \multicolumn{5}{|c|}{ Liver transplantation } \\
\hline \multirow{3}{*}{\multicolumn{2}{|c|}{ Overall }} & No & Yes & \multirow{4}{*}{ P-value } \\
\hline & & \multirow{3}{*}{$\begin{array}{c}n=5526 \\
\text { weighted } n=27266 \\
N(w t \%)\end{array}$} & \multirow{3}{*}{$\begin{array}{c}n=73 \\
\text { weighted } n=365 \\
N(w t \%)\end{array}$} & \\
\hline & & & & \\
\hline Outcomes & N (wt \%) & & & \\
\hline \multicolumn{5}{|c|}{ Death or AMA } \\
\hline No & $5273(94.17)$ & $5201(94.1)$ & $72(99.15)$ & \multirow{2}{*}{0.21} \\
\hline Yes & $326(5.83)$ & $325(5.9)$ & $1(0.85)$ & \\
\hline \multicolumn{5}{|c|}{ Organ failure } \\
\hline No & 5140 (91.84) & $5070(91.8)$ & $70(95.46)$ & \multirow{2}{*}{0.291} \\
\hline Yes & $459(8.16)$ & $456(8.2)$ & $3(4.54)$ & \\
\hline \multicolumn{5}{|c|}{ subjects with acute pancreatitis. } \\
\hline \multicolumn{5}{|c|}{ AMA: Against Medical Advice; WT: Weighted. } \\
\hline \multicolumn{5}{|c|}{ Significant values in bold $(P<0.05)$} \\
\hline
\end{tabular}

\section{Discussion}

Results of the present study show that AP patient with hepatitis virus infection have significantly increased odds of death or leaving hospital AMA and organ failure compared to AP patients without hepatitis virus infection. Significant differences were also found in number of deaths or AMA between hepatitis virus-infected patients who underwent liver transplantation compared to those without transplantation, although no significant differences were found in organ failure between hepatitis virus-infected patients with or without liver transplantation. In addition, no significant associations were found between the presence of hepatitis virus infection in AP patients and colonic necrosis, splenic vein/portal vein thrombosis or hospital LOS. Furthermore, AP patients with HCV had significantly higher odds of death or AMA, and organ failure. In contrast, AP patients with HBV had significantly increased odds of organ failure only.

Previous studies have also reported increased deaths and organ failure in AP patients with viral hepatitis. Viruses are, in fact, the largest group of infectious organisms associated with AP, and HBV is most often the causative hepatitis virus, especially in those who have undergone liver transplantation [4,5]. In a meta-analysis of AP patients, risk of death was found to double when severity was marked by the presence of infected pancreatic necrosis and organ failure; when either is present, the diagnosis is critical AP and when both are present the mortality rate is as high as $43 \%[13,16]$. In AP that develops in conjunction with fulminant viral hepatitis, mortality is contingent upon severity of the hepatitis infection; while AP with HAV responds to conservative measures, HCV and HEV are associated with necrotizing pancreatitis, multiple organ failure and death $[14,17]$.
In the present study, hepatitis virus-infected AP patients were significantly more likely to leave the hospital AMA than those without viral hepatitis. However, since NIS data used in this study were discharge data without follow-up, individual readmission statistics were not available. Also, because AMA substantially increases mortality and readmission $[15,16,18,19]$, we combined the AP outcomes of death and AMA. The adverse consequences of leaving the hospital AMA are evidenced in higher rates of 90-day mortality and 30-day readmission of 21,400 patients discharged AMA among nearly 2 million alive patients discharged [16,19]. Also, AMA patients are often readmitted in critical condition and more likely to have organ failure leading to death $[15,16,18,19]$. Post-discharge interventions have been suggested for the $1 \%$ to $2 \%$ of patients leaving AMA to help reduce the higher risk of adverse outcomes $[15,18]$.

Colonic necrosis and splenic vein thrombosis are noted complications of AP $[17,18,20,21]$ and colonic involvement especially is associated with increased mortality due to erosion or inflammation of the large bowel and progression to life threatening necrosis, hemorrhage, or perforation $[17,20]$. The predictive factors identified for splenic vein thrombosis in severe AP are alcoholism, smoking and male gender; however, in that study, viral causes were listed with other possible causes of AP but were not associated with severe AP [18,21]. Other authors have also reported colonic stenosis following severe AP [19,20,22,23] Aswani et al. [20,23] reviewed venous complications of $\mathrm{AP}$, noting that splenic vein thrombosis may lead to splenic complications such as rupture or subscapular hematoma, and that in portal vein thrombosis, cavernous transformation of the portal vein occurring within three weeks after obstruction is expected to maintain hepatoportal flow in about $70 \%-100 \%$ of patients. In the present study, however, no 
significant associations were found between colonic necrosis or splenic vein thrombosis in AP patients with hepatitis virus infection compared to those without viral hepatitis.

Among the viral hepatitis subtypes in the present study, HCV was predominant with $3 \%$ of AP patients in the NIS database population, followed by HBV and HAV. In the United States, hepatitis virus incidence rates are highest for HBV, followed by HCV and HAV $[21,24]$, so the NIS database does not appear to be representative. The lower incidence rates in the NIS may be attributed to inactive HBV carriers unaware of their positive HBV status at the time of hospitalization. Inactive carriers are the largest group (300 million) among patients infected with HBV, and the diagnostic workup can mask positive HBV status because it is based on negative HBeAg and positive anti-HBeAg, with normal Alanine Amino Transferase (ALT) levels and only slight liver fibrosis [21,24]. In a study of the frequency and characteristics of pancreatitis in acute viral hepatitis, HAV and HBV were present among $5 \%$ of patients with AP, and a small percentage had HEV [11]. While none of the hepatitis virusinfected AP patients in the NIS database had HEV, at least 14 case studies of HEV infection associated with AP have been reported in the literature, mainly describing young adults in areas endemic for the virus $[22,23,25,26]$. Non-fulminate acute hepatitis E virus HEV infection complicated a moderately severe AP in a 7-year-old boy who also had Glucose 6 Phosphate Dehydrogenase (G6PD) deficiency [22,25].

A review study identified 55 cases of HEV-associated AP, among which about one-fifth were severe and overall mortality was 3.2\% [24,27] Kamar et al. [25,28] reported that HEV, besides its association with AP, was also associated with extra hepatic disorders, including hematologic disorders and autoimmune myocarditis and thyroiditis. In the present study, significant differences were found in the severity of illness between hepatitis virus subtypes. Severity was determined in the NIS database by All Patient Refined-Diagnosis Related Group (APR-DRG/ Severity), a risk of mortality score used to determine severity (ranked as minor, moderated, major, and extreme) in the ICU setting [26,29]. The APR-DRG severity score system is used to evaluate not just AP but the overall condition of medical ICU patients, making especially useful for distinguishing severity levels between hepatitis subtypes. Accordingly, in our evaluation of severity of illness, severity refers not to either AP or viral hepatitis but to patients' overall condition, especially since "overall" severity is a better indicator when multiple organs may be involved $[27,30]$.

Liver transplantation in 73 hepatitis virus-infected patients complicated AP in the present study, and significant associations were found between death or AMA and liver transplantation. The transplantation group had different hepatitis virus subtypes: one patient had HAV, 7 had HBV and 65 had HCV. AP has been reported as a side effect of Paginated Interferon (PEG-IFN) and Ribavirin (RBV) therapy administered to treat viral hepatitis infection in liver transplant patients $[13,14,28,29]$. After observing three patients with AP after PEG-IFN therapy combined with RBV, Ando et al. $[29,14]$ concluded that stimulation of the immune system by the combination therapy may have caused the AP. Animal studies, however, have suggested that interferon induces AP rather than ribavirin [30,31]. Other drugs (sofosbuvir, simeprevir) used to treat an aggressive form of HCV infection (fibrosing cholestatic hepatitis) that develops in association with liver transplant, led to development of AP and refractory purities [31,32]. The previous literatures showed that AP was closely related to HCV therapy; however, we found AP is associated with HCV infection in this study (Table 6). This is a new finding that need to be verified by more evidence and studies. To further explore whether the liver transplantation will be activated or promote HCV infection in AP patients is also needed.

A comprehensive review found that re-transplant was needed in about $25 \%$ of cases of post-liver transplant AP [32,33]. Per transplant pancreatitis, arising after liver transplantation was suggested to serve as a marker of the high mortality and graft failure occurring in liver transplant patients [33,34]. Nevertheless, guidelines are not available for the treatment of liver transplantassociated AP in hepatitis virus-infected patients and studies focusing on risk factors are needed to help reduce the significant morbidity and mortality associated with post-transplant AP.

\section{Strengths and Limitations}

An important part of our strategy for this study was using the comprehensive, nationally representative patient data in the 20052014 National Inpatient Sample, the largest inpatient sample in the United States containing data from more than 1,000 participating hospitals in the United States was included.

a. Study variables were identified based on medical records of inpatient admissions using ICD-9 diagnostic codes from discharge data. Therefore, the time sequence of individual exposure to hepatitis virus infection and outcomes is not totally clear, that is, it is not known in every case whether the hepatitis virus was active during hospitalization.

b. Additionally, NIS is a cross-sectional dataset and data were analyzed retrospectively, which precludes inferring causality and limits data interpretation. Although ICD-9 diagnosis codes were used, they depend on the accuracy of hospital coders who assign the codes based on discharge data and pathology reports. Nevertheless, errors in ICD coding are reported to be limited [34-37].

c. Also, Unmeasured confounders such as lifestyle, behavior, environmental exposure, family medical history and clinical lab 
data were not included in the NIS database and could not be accounted for.

d. Because the NIS database includes discharge data only, the present study also could not evaluate long-term health status of the patient population. Long-term prospective study is required to confirm results of the present study and to further explore associations between AP outcomes in the presence of acute and chronic viral hepatitis.

\section{Conclusion}

In conclusions, AP patients with hepatitis virus infection have significantly increased odds of death or leaving hospital AMA and organ failure compared to those without hepatitis virus infection. Deaths or AMA are significantly different between hepatitis virusinfected patients receiving liver transplantation and those without transplant, but no differences are found in organ failure. Significant differences are found in severity of illness between hepatitis virus subtypes. Results of this study help to ascertain the impact of different hepatitis subtypes on AP prognosis and may help to develop strategies for clinical practice by targeting anti-viral agents that can ameliorate AP effectively.

\section{Ethical Committee Approval}

Because the NIS originally received signed informed consent from all included patients to participate in data collection for later evaluation, and patient data in the NIS database were subsequently deidentified, signed informed consent was waived for the present study.

\section{Acknowledgements}

None

\section{Funding}

None

\section{References}

1. Roberts SE, Akbari A, Thorne K, Atkinson M, Evans PA (2013) The incidence of acute pancreatitis: impact of social deprivation, alcohol consumption, seasonal and demographic factors. Aliment Pharmacol Ther 38(5): 539-548.

2. Forsmark CE, Vege SS, Wilcox CM (2016) Acute pancreatitis. N Engl J Med 375(20): 1972-1981.

3. Kaurich T (2008) Drug-induced acute pancreatitis. Proceedings (Baylor University Med Ctr) 21(1): 77-81.

4. Rawla P, Bandaru SS, Vellipuram AR (2017) Review of infectious etiology of acute pancreatitis. Gastroenterology Res 10(3): 153-158.

5. Economou M, Zissis M (2000) Infectious cases of acute pancreatitis. Ann Gastroenterol 13: 98-101.

6. Zuckerman AJ (1996) Hepatitis Viruses. In: Baron S, editor, Medical Microbiology, 4th ed. Galveston, TX: University of Texas Ch. 70 USA
7. Wasley A, Grytdal S, Gallagher K, Centers for Disease Control and Prevention (2006) Surveillance for acute viral hepatitis-United States. MMWR Surveillance Summary 58: 1-27.

8. Centers for Disease Control and Prevention (2014) Viral Hepatitis Surveillance United States.

9. Khedmat H, Ghamar-Chehreh ME, Agah S, Aghaei A (2015) Pancreatitis developing in the context of acute hepatitis: A literature review. J Pancreas 16(2): 104-109.

10. Sass DA, Shakil AO (2005) Fulminant hepatic failure. Liver Transpl 11: 594-605.

11. Jain P, Nijhawan S, Rai RR, Nepalia S, Mathur A (2007) Acute pancreatitis in acute viral hepatitis. World J Gastroenterol 13: 5741-5744.

12. Haffar S, Bazerbachi F, Prokop L, Watt KD, Murad MH, et al. (2017) Frequency, and prognosis of acute pancreatitis associated with fulminant or non-fulminant acute hepatitis A: A systematic review. Pancreatotomy 17(2): 166-175.

13. Choi JW, Lee JS, Paik WH, Song TJ, Kim JW, et al. (2016) Acute pancreatitis associated with pegylated interferon-alfa-2a therapy in chronic hepatitis C. Clin Mol Hepatol 22(1): 168-171.

14. Ando K, Kim SR, Imoto S, Nakajima T, Mita K, et al. (2009) Acute pancreatitis associated with pegylaated interferon and ribavirin treatment of chronic hepatitis $C$, genotype $1 \mathrm{~b}$ with high viral load. Case Rep Gastroenterol 3(3): 372-376.

15. (2017) Overview of the Nationwide Inpatient Sample (NIS).

16. Petrov MS, Shanbhag S, Chakraborty M, Phillips AR, Windsor JA (2010) Organ failure and infection of pancreatic necrosis as determinants of mortality in patients with acute pancreatitis. Gastroenterol 139(3): 813820.

17. Haffar S, Bazerbachi F, Prokop L, Watt KD, Murad MH, et al. (2017) Frequency, and prognosis of acute pancreatitis associated with fulminant or non-fulminant acute hepatitis A: A systematic review. Pancreatol 17(2): 166-175.

18. Glasgow JM, Baughn-Sarrazin M, Kaboli PJ (2010) Leaving Against Medical Advice (AMA): risk of 30-day mortality and hospital readmission. J Gen Intern Med 25(9): 926-929.

19. Garland A, Ramsey CD, Fransoo R, Olafson K, Chateau D, et al. (2013) Rates of readmission and death associated with leaving hospital against medical advice: a population-based study. CMAJ 185(14): 1207-1214.

20. Nagpal AP, Soni H, Haribhakti S (2015) Severe colonic complications requiring total colectomy in acute necrotizing pancreatitis-A retrospective study of 8 patients. Indian J Surg 77(1): 3-6.

21. Toqué L, Hamy A, Hamel JF, Cesbron E, Hulo P, et al. (2015) Predictive factors of splanchnic vein thrombosis in acute pancreatitis: A 6-year single-center experience. J Dig Dis 16(12): 734-740.

22. Maisonnette F, Abita T, Pichon N, Lachachi F, Cessot F, et al. (2003) Development of colonic stenosis following severe acute pancreatitis. HPB (Oxford) 5(3): 183-185.

23. Aswani Y, Hira P (2015) Venous complications of pancreatitis: a review. JOP 16(1): 20-24.

24. Sharma SK, Saini H, Chwla Y (2005) Hepatitis B virus: inactive carriers. Virol J 2: 82 .

25. Thapa R, Biswas B, Mallick D, Ghosh A (2009) Acute pancreatitiscomplicating hepatitis E virus infection in a 7-year-old boy with glucose 6 phosphate dehydrogenase deficiency. Clin Pediatr (Phila) 48(2): 199201. 
26. Raj M, Kumar K, Ghoshal UC, Saraswat VA, Aggarwal R, et al. (2015) Acute hepatitis E-associated acute pancreatitis: A single center experience and literature review. Pancreas 44(8): 1320-1322.

27. Haffar S, Bazerbachi F, Garg S, Lake JR, Freeman ML (2015) Frequency, and prognosis of acute pancreatitis associated with acute hepatitis E: A systematic review. Pancreatol 15(4): 321-326.

28. Kamar N, Marion O, Abravanel F, Izopet J, Dalton HR (2016) Extrahepatic manifestations of hepatitis E virus. Liver Int 36(4): 467-472.

29. Baram D, Daroowalla F, Garcia R, Zhang G, Chen JJ, et al. (2008) Use of the All Patient Refined-Diagnosis Related Group (APR-DRG) risk of mortality score as a severity adjustor in the medical ICU. Clin med Circ Respirat Pulm Med 2: 19-25.

30. Banks PA, Bollen TL, Dervenis C, Gooszen HG, Johnson CD, et al. (2013) Classification of acute pancreatitis-2012: revision of the Atlanta classification and definitions by international consensus. Gut 62: 102111.

31. Choi JW, Lee JS, Paik WH, Song TJ, Kim JW, et al. Acute pancreatitis associated with pegylated interferon-alfa-2a therapy in chronic hepatitis C. Clin Mol Hepatol 22(1): 168-171.
32. Ando K, Kim SR, Imoto S, Nakajima T, Mita K, et al. (2009) Acute pancreatitis associated with pegylated interferon and ribavirin treatment of chronic hepatitis $C$, genotype $1 \mathrm{~b}$ with high viral load. Case Rep Gastroenterol 3(3): 372-376.

33. Roncal-Jimenez CA, Lanaspa MA, Rivard CJ, Nakagawa T, Sanchez-Lozada LG, et al. (2011) Sucrose induces fatty liver and pancreatic inflammation in male breeder rats independent of excess energy intake. Metabolism 60(9): 1259-1270.

34. Issa D, Eghtesad B, Zein NN, Yerian L, Cruise M, et al. (2016) Sofosbuvir and simeprevir for the treatment of recurrent hepatitis $\mathrm{C}$ with fibrosing cholestatic hepatitis after liver transplantation. Int J Organ Transplant Med 7(1): 38-45.

35. Danalığlu A, Mitchell OJ, Singh VK, Danalığlu AN, Sentürk H, et al. (2015) Acute pancreatitis following adult liver transplantation: A systematic review. Turk J Gastroentrol 26(6): 450-455.

36. Russell TA, Park S, Agopian VG, Zarrinpar A, Farmer DG, et al. (2017) Peri transplant pancreatitis A marker for high mortality and graft failure in liver transplant patients. Liver Transpl 23(7): 925-932.

37. (2017) Report of the National Inpatient Sample of the Healthcare Cost and Utilization Project. 